e n supposant l'excentricité $=0,046687$. Les équations de condition m'ont donné la correction de l'équation du centre - 9"7, $=2 d e$, quantité que j'ai retranché du 1 ex terme seulement, sans corriger les autres termes de l'équation du centre. J'ai oublié de corriger la formule de la page $X V$. La formule de l'équation du centre après les corrections sera,

$$
\begin{aligned}
& \text { +59417,84 } \operatorname{Sin}\left(\varphi^{\prime \prime}-\varpi^{\prime \prime}\right) \\
& +1732,58 \operatorname{Sin} 2\left(\varphi^{\prime \prime}-\varpi^{\prime \prime}\right) \\
& +\quad 70,06 \operatorname{Sin} 3\left(\varphi^{\prime \prime}-\varpi^{\prime \prime}\right) \\
& +\quad 3,25 \operatorname{Sin} 4\left(\varphi^{\prime \prime}-\varpi^{\prime \prime}\right) \\
& +\quad 0,16 \operatorname{Sin} 5\left(\varphi^{\prime \prime}-\varpi^{\prime \prime}\right)
\end{aligned}
$$

Les équations de condition ayant donné la correction de l'excentricité $d e=-4^{\prime \prime}, 85$ on en partie de l'unité $=-0,00000762$, quantilé à retrancher de l'excentricité $e=0,046687$, donnent pour l'excentricité act uelle $e=0,04667938$, mais en fuisant cette reduclion je me suis trompé, en supposant la correction ci-dessus, dix fois trop grande: ce qui justifie complettement la remarque de Mr. Bessel.
Le table XII d'Uranus, dépendante de l'argument $\varphi^{\prime}-2 \varphi^{\prime \prime}$, n'est pas exacte, elle est trop forte de $1^{\prime \prime}, 3$. N'ayant pas relrouvé mes calculs originaux, j'ai repris les formules de la mécanique Célesie Tom. III, page 145. En réunissant les deux termes, et en tenant compte de la reduction de la masse de Saturne, j'ai trouvé pour 1800, la fornule

$$
-435^{\prime \prime}, 12 \operatorname{Sin}\left(\varphi^{\prime}-2 \varphi^{\prime \prime}+80^{\circ}, 13\right)
$$

Elle ne s'accorde pas parfaitement avec celle que jai employé pour construire la XII, mais heureusement 1'erreur est insensible.

Dans la page II de l'introduction, j'ai donné les longitudes des périhelies et des noeuds, par rapport au l'équinoxe; mais j'ai encore oublié d'ajouter la précéssion $154^{\prime \prime}, 63 t$ au perihélie des deux premières planètes: Jupiter et Saturne.

Voila Monsieur, les renseignemens que je puis Vous donner, concernant mes tables d'Uranus.

$$
B \text { o u } v a r d
$$

\title{
Auszug aus einem Briefe des Herrn Bouvard an den Herausgeber.
} Paris 1823. Dec. 13.

J'ai le plaisir de Vous envoyer les observations de la Lnne et des petifes étoiles qui ont été observées pour déterminer la différence des Méridiens. Elles ne sont pas très nombreuses parceque les circonstances atmosphériques n'ont pas permis d'en faire d'avantage, car le ciel de Paris n'est pas très favorable aux observations astronomiques. Parmis les étoiles qui peuvent être comparées à la lnne, j'ai mis dans le tableau suivant quelques unes qui ne sont pas indiquées; mais j'ai pensé qu'elles pourxaient être utiles. La copie ci-jointe de nos observations a éé collationnée avec le plus grand soin, je vous prie en conséquence, Monsieur, de vouloir bien en surveiller limpression.

Daus des momens de loisir, je cumpare nos observations avec celles que vous donnez dans votre journal astronomique; mais en me servant de ces observations, j'ai reconnu un assez grand nombre d'observations inexactes.

La $2^{e}$ étoile du 11 avril 1821 observées à Dorpat et la 2 e du 15 mai de la même année sont fausses, đinsi que la 309 Mayer, 3 mars 1822.

7 mars 1822, à Coppenhague, 212 page 169 est fautive,

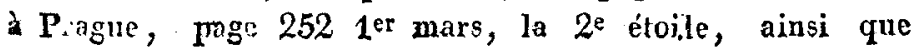

la $2^{\text {e }} 4$ mars sont érronnées. Comme je n'ai pas achevé mes calculs j'ignore s'il existe d'autres.

J'ai également vî avec satisfaction, dang le dernier No. de votre journal, les observations faites à Paramatta, par MM. Brisbane et Rümker. Les mêmes observations m'ont été adressées. La lettre de mon ami, le gouverneur, est datée du 16 avril dernier; elle ne m'est parvenue que vers le 16 Novembre dernier. Mr. le Major-Général me mande, qu'ils ont déjà observée dix mille étoiles de l'hémisphère austral et que les reductions sont très avancées; que Mr. Rümker élant d'une si grande activité tant pour les observations, que pour les calculs, ce travail sera bientôt termińé. Il m'annonce aussi qu'il reste encore environ dix mille étoiles à déterminer avant d'avoir epuisé cetle mêrne partie du ciel invisible en Europe; et que dans 10 mois, ces observations, seront très probablement terminées.

Comme Mr. le gonverneur désire beaucoup de trouver des observations correspondantes, je crois devoir Vous adresser celles qui ont été faites à Paris. Voici les observalions: 
27 Mai 1822. Lune $=10^{\text {h }} 25^{\prime} 44^{\prime \prime}, 06$; Réguhus $=9^{\text {h }} 57^{\prime} 58^{\prime \prime}, 54$ $=27^{\prime} 4 j^{\prime \prime}, 52$. Corrigé de la marche de la pendule: différence des méridicns $9^{\mathrm{h}} 54^{\prime} 42^{\prime \prime}, 3$.

29 Aout 1822. Lune $=20^{\mathrm{h}} 26^{\prime} 13^{\prime \prime}, 60 ; 4$ Capr. $=20^{\mathrm{h}} 7^{\prime} 7^{\prime \prime}, 86$, en reduisant celte étoile à $v$ Capricorne observée Paramalla, j'ai trouvé la différence des méridiens $9^{\text {in }} 54^{\prime} 46^{\prime \prime}, 8$.

Observations de la Luns, faites ì l'observatoire Royal de Paris, pour la détermination de la différence des méridiens.

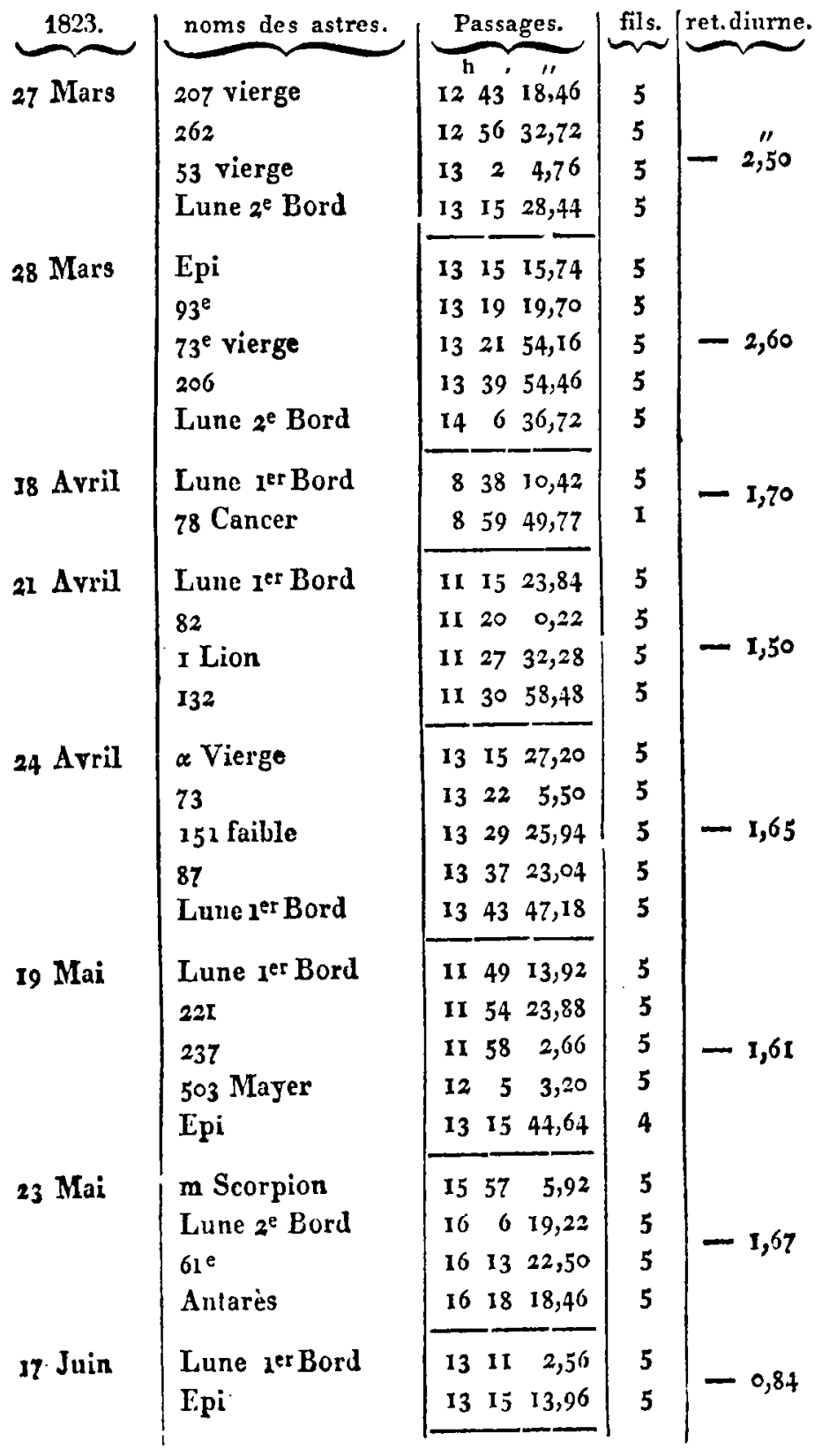

$$
17
$$

17 Juillel

Lune Ier Bord

\& Scorpion

Antarès

20 Juillet Lune $1^{\text {er Bord }}$

$\lambda$ Sagitt.

$24^{\mathrm{e}}$

155 e

24 Juillet

I8 Verseau

885

Lune $2^{\mathrm{e}}$ Bord

$\propto$ Verseau

16 Aûut

Lune Ier Bord

383

$\lambda$ Sagitt.

20 Aôut

9 Verseau

872 Mayer.

92 Capric.

Lune I ${ }^{\text {er Bord }}$

13 Sept. Lune rer Bord

$\varphi$ Sagiltair

I9l

$v$ Sagitt.

16 Sept. Lune $1^{\text {er Bord ond. }}$

877 Mayer

18 Verseau

144

18 Sept. Lune ${ }_{1}^{\text {er Bord }}$

219

242

80 Verscau

19 Sept. Lune yer Bord

16 poissons

$\lambda$ id.

383

20 Sept. 979 Mayer

26 poissons

$\omega$ id.

Lune $2^{\mathrm{e}}$ Bord

II Oct. Lune $x^{e r B o r d}$ nuag.

$77^{1}$ Sagitt. Mayer.

If Oct. $59^{\mathrm{e} \text { Verseau }}$

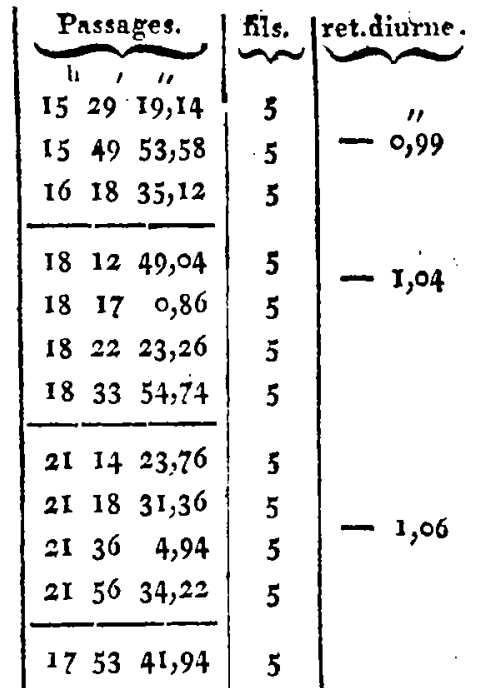

$-1,30$

$\begin{array}{lll}17 & 39 & 34,18 \\ 18 & 16 & 29,82\end{array}$

$20 \quad 5043,22$

$\begin{array}{lll}21 & 1 & 14: 98\end{array}$

$\begin{array}{lll}21 & 5 & 17,50\end{array}$

$\begin{array}{llll}21 & 17 & 42,92\end{array}$

$\begin{array}{llll}18 & 27 & 34,08\end{array}$

$18 \quad 34 \quad 16,32$

$\begin{array}{lll}18 & 39 & 16,58\end{array}$

$1847 \quad 5,22$

$2059 \quad 4 \%, 30$

$\begin{array}{lll}21 & 8 & 59,38\end{array}$

$2117 \quad 5,26$

$21 \quad 1933,06$

$2233 \quad 48,26$

$\begin{array}{lll}22 & 38 & 13\end{array}$

$22 \quad 44 \quad 4,50$

$22 \quad 51 \quad 52,50$

$2320 \quad 27,70$

$23 \quad 26 \quad 50,18$

$\begin{array}{lll}23 & 32 & 29,62\end{array}$

$\begin{array}{lll}23 & 36 & 59,76\end{array}$

233513,76

$23 \quad 45 \quad 31,22$

$23 \quad 50 \quad 17,10$

o $10 \quad 15,80$

185911,74

$\frac{19}{21} \frac{436,00}{835,64}$ $3 I^{\star}$ 


\begin{tabular}{|c|c|}
\hline 1823. & Noms des astre \\
\hline 14 Oct. & $\begin{array}{l}18 \text { Verseall } \\
885 \text { Nayer } \\
\text { Lune te Bord }\end{array}$ \\
\hline 18 Oct. & $\begin{array}{l}88 \text { poissons } \\
85 \\
110 \\
\text { Lune } I^{e} \text { Bord }\end{array}$ \\
\hline${ }_{5}$ Oct. & $\begin{array}{l}30 \text { Verseau } \\
\alpha \text { Verseau } \\
36 \text { id. } \\
\text { Lune }{ }_{1}^{\text {er Bord }}\end{array}$ \\
\hline 19 Oct. & $\begin{array}{ll}311 & \text { poissons } \\
8 ? \quad \text { Id. } \\
\eta & \text { id. } \\
\text { Lune } & \text { er } \\
\text { Bord }\end{array}$ \\
\hline 20 Oct. & $\begin{array}{l}y \text { Belier } \\
v \text { id. } \\
26 \\
\text { Lune } z^{\text {e Bord }}\end{array}$ \\
\hline Io Nov. & $\begin{array}{l}\text { Lune } 1 \text { er Bord } \\
18 \text { Verseau } \\
144 \\
177\end{array}$ \\
\hline
\end{tabular}

\begin{tabular}{|c|c|c|}
\hline Passages. & fils. & |ret. diurne \\
\hline 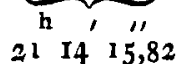 & & \\
\hline $21 \quad 18 \quad 23,38$ & 5 & - I,82 \\
\hline $\begin{array}{lll}21 & 27 & 33,28\end{array}$ & 5 & \\
\hline o 756,96 & 5 & \\
\hline ㅇ $20=25,26$ & 5 & - T \\
\hline ० 2439,86 & 5 & 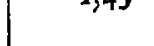 \\
\hline - $3725,3^{\circ}$ & 5 & \\
\hline $21 \quad 53 \quad 41,18$ & 5 & \\
\hline $\begin{array}{lll}21 & 56 & 24,98\end{array}$ & 5 & -0 \\
\hline $21 \cdot 59 \quad 49,84$ & 5 & $-1,80$ \\
\hline $22 \quad 14 \quad 11,34$ & 5 & \\
\hline $1 \circ 27,08$ & 8 & \\
\hline 1422,18 & 5 & \\
\hline $\begin{array}{lll}121 & 39,48\end{array}$ & 5 & $-1,45$ \\
\hline 12943,02 & 5 & \\
\hline 2231,00 & 5 & \\
\hline 2754,30 & 5 & \\
\hline 22020,18 & 5 & $-1,51$ \\
\hline $228 \quad 9,14$ & 5 & \\
\hline $21 \quad 7 \quad 45,38$ & 5 & \\
\hline 211440,60 & 5 & $-1,07$ \\
\hline $21 \quad 20 \quad 7,90$ & 5 & \\
\hline $212+46,76$ & 5 & \\
\hline
\end{tabular}

\begin{tabular}{|c|c|c|c|c|}
\hline$\underbrace{1823 .}$ & Noms des astres. & $\underbrace{\text { Passages. }}$ & $\underbrace{\text { fils. }}$ & $\underbrace{\text { ret. diurne. }}$ \\
\hline \multirow[t]{4}{*}{11 Nor. } & Lune $1^{\text {er Bord }}$ & $2154^{\prime} 8,82$ & 5 & \\
\hline & 919 Mayer & 22740,56 & 5 & - \\
\hline & & $\begin{array}{lll}22 & 14 & 24,24\end{array}$ & 5 & \\
\hline & $\sigma$ Verseau & $2221 \quad 25,84$ & 5 & \\
\hline \multirow[t]{4}{*}{${ }_{3} 3$ Nov. } & $x$ poissons & $23 \quad 17 \quad 59,48$ & 5 & \\
\hline & Lune yer Bord & $23 \quad 26 \quad 14,34$ & 5 & $-0,77$ \\
\hline & $\lambda$ poissons & $\begin{array}{lll}23 & 33 & 9,08\end{array}$ & 5 & \\
\hline & 183 & $23 \quad 37 \quad 38,96$ & 5 & \\
\hline \multirow[t]{4}{*}{16 Nov. } & 4 Belier & I $3841,5^{\circ}$ & 5 & \\
\hline & $8 \mathrm{i}$ id. & $147 \quad 47,42$ & 5 & $-0,92$ \\
\hline & & 1546,46 & 5 & \\
\hline & Lune 1 er Bord & $159 \quad 23.32$ & 5 & \\
\hline \multirow[t]{4}{*}{17 Nuv. } & Lune ${ }_{1}$ er Bord & $2 5 8 \longdiv { 3 8 , 8 6 }$ & 5 & \\
\hline & I 1 Belier & 3116,74 & 5 & -0.01 \\
\hline & 86 & $\begin{array}{lll}3 & 18 & 12,16\end{array}$ & 5 & $0,9+$ \\
\hline & 128 & 33416,44 & 5 & \\
\hline \multirow[t]{4}{*}{10 Dec. } & Lune yer Bord & 23535,82 & 5 & \\
\hline & 962 Mayer & 231422,76 & 5 & $+0,05$ \\
\hline & 12 Poissons & 232021,52 & 5 & \\
\hline & ${ }_{4} 4$ Poissons & $23 \quad 27 \quad 58,74$ & 5 & \\
\hline
\end{tabular}

Le tems des passages des éloiles sont cemx qui ont élé observés, sans ancune correclion. Pour tenir comple du retard de la pendule on l'a donné pour chaque jour d'observation; il sera donc facile d'y avoir égard dans le calcul.

$$
B \circ u v a r d
$$

Trigonometrische Ortsbestimmungen in Westphalen, aus Privatmessungen des Herrn Professors Oltmanns.

Die Resultate einer trigonometrischen Mressung, welche ich für lhre astronomischen Nachrichten ïhersende, kann ich mcine eigenen nemnen. Denn, ohne anderweite Plichten zu verabsäumen, bliei mir so viel Mrusse ïbrig, ura mit einem kleinen Mulliplications-Kreise terrestrische Winkel in jenen Gegenden zu messen, wo, $25 \mathrm{Jahl} r$ früher, z'on Lecoq mit seinen hoch yerdienten Offizieren des GeneralStabs gearbeitet halte.

Die Verniere vorbenannten Kreises gaben $15^{N}$ an; was ihm an grölserer Genauigkeit abging, suchte ich durch pnssende Combinalionen der Dreyecke zu ersetzen. Ich glaube, die bey Kataster-'Triangulirungen vorgeschriebene Utnauigkeit nicht allein erreicht, sondern auch ïberiroffen zu hahsen, und die Uebereinslimmung mit Krayenhoys Dreyecken scheint dem nichl zu widersprechen:
Die Längen und Breiten sind in der Alpplattung von $\frac{\pi}{3} 4$ nach Bolnenberger's Formeln berechnet worden. Einige friiber bekannt gemachte Resultate haben deswegen cine Aenderung in den geographischen Breiten erlitten,

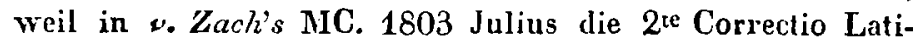
tudinis mit verkehrtem Zeichen angebracht wird. Und diese beträgt bey Rheine $6^{\prime \prime}, 2$.

Ueber die geringe Anzahl der Resultate brauche ich mich eben nicht zu entschuldigen. Die wustphälischen Sleppen bieten wenig Visirbares dar. Von der ostfricsisehen Grenze bis an die prenfsisclie trifft man oft auf 2 bis 3 Stunden Weges keine menschliche Wohnung an. Auch das Klima bietel eigenthümliche Schwicrigkeilen dar. Im Frühling und Herbste: nix, nox el nebulae scmper. In Sommer ewiger Moordampf, der nicht vergünnt, 